\title{
APPROXIMATION OF ARITHMETICAL FUNCTIONS BY ADDITIVE ONES
}

\author{
JANOS GALAMBOS
}

Abstract. Let $\varepsilon_{p}(n)=1$ or 0 according as $p \mid n$ or not. Since the functions $\varepsilon_{p}(n)-1 / p$ are quasi-orthogonal on the integers 1 , $2, \cdots, N$ with the relative frequency as measure, the theory of orthogonal expansions suggests an approximation of arbitrary arithmetical functions by strongly additive ones. In the present note, the approximating additive functions are determined and a sufficient condition is given for an arithmetical function to have an asymptotic distribution. Examples are given to illustrate the result.

1. Determination of the approximating additive functions. Let $\varepsilon_{p}(n)=1$ or 0 according as $p \mid n$ or not, where $p$ is a given prime number. Evidently

$$
N^{-1} \sum_{n=1}^{N} \varepsilon_{p}(n)=N^{-1}[N / p]=1 / p+O(1 / N),
$$

where $[y]$ signifies the integer part of $y$. Thus the mean value of $\varepsilon_{p}(n)-1 / p$ is asymptotically zero. Since for $p \neq q$,

$$
\begin{aligned}
N^{-1} \sum_{n=1}^{N}\left\{\varepsilon_{p}(n)-1 / p\right\}\left\{\varepsilon_{q}(n)-1 / q\right\} \\
=\frac{1}{N}\left[\frac{N}{p q}\right]-\frac{1}{N p}\left[\frac{N}{q}\right]-\frac{1}{N q}\left[\frac{N}{p}\right]+\frac{1}{p q}=O\left(\frac{1}{N}\right),
\end{aligned}
$$

the sequence $\left\{\varepsilon_{p}(n)-1 / p\right\}$ is quasi-orthogonal as $p$ runs through the prime numbers. This suggests that a "good" approximation to an arbitrary arithmetical function $f(n)$ can be found in the form

$$
F_{N}(f, n)=c_{1}(f)+\sum_{p \leqq N} c_{p}(f) \varphi_{p}(n),
$$

where $\varphi_{p}(n)=a(p)\left\{\varepsilon_{p}(n)-1 / p\right\}$, suitably normalized to achieve

$$
N^{-1} \sum_{n=1}^{N} \varphi_{p}^{2}(n) \sim 1 \quad(N \rightarrow+\infty)
$$

Presented in part to the Society, January 25, 1972 under the title Additive functions as quasi-orthogonal series for arithmetical functions; received by the editors July 6, 1972.

AMS (MOS) subject classifications (1970). Primary 10K20; Secondary 10L10, 10A20, $10 \mathrm{H} 25$.

Key words and phrases. Arithmetical functions, additive, multiplicative, limiting distribution, asymptotic mean value, approximation, quasi-orthogonal functions, Erdös theorem, Turán-Kubilius inequality.

(c) American Mathematical Society 1973 
and where the coefficients $c_{p}(f)$ are constants depending on $f(n)$ and $p$, but not on $n$ itself. Note that the functional part

$$
G_{N}(f, n)=\sum_{p \leqq N} c_{p}(f) a(p) \varepsilon_{p}(n)
$$

of (3) is a strongly additive function. We recall that $g(n)$ is strongly additive if, for $(u, v)=1$,

$$
g(u, v)=g(u)+g(v)
$$

and $g\left(p^{r}\right)=g(p)$ for all primes $p$ and integers $r \geqq 1$. In order to satisfy (4), we specify $a(p)$ as

$$
a(p)=p /(p-1)^{1 / 2}
$$

and thus

$$
\varphi_{p}(n)=p(p-1)^{-1 / 2}\left\{\varepsilon_{p}(n)-1 / p\right\} .
$$

(7) is obtained from (1) by taking asymptotic values. As a matter of fact, since

$$
\left(\varepsilon_{p}(n)-1 / p\right)^{2}=\varepsilon_{p}(n)-2 \varepsilon_{p}(n) / p+1 / p^{2},
$$

its asymptotic mean value by $(1)$ is $p^{-1}(1-1 / p)$, hence, by the choice in (7), (4) is satisfied. The choice of the coefficients $c_{p}(f)$, on the other hand, depends on, and is determined by, the concept of "good" approximation. We shall select our approximating function $F_{N}(f, n)$ so that it asymptotically be the closest to $f(n)$ in mean squares. More precisely, putting

$$
M(g(n), N)=N^{-1} \sum_{n=1}^{N} g(n),
$$

our aim is to determine the coefficients $c_{p}(f)$ in (3) by (asymptotically) minimizing

$$
d\left(f, F_{N}\right)=M\left(\left\{f(n)-F_{N}(f, n)\right\}^{2}, N\right) .
$$

This choice of the coefficients $c_{p}(f)$ will fully be exploited in the next section. If the sequence $\varphi_{p}(n)$ were exactly orthogonal, i.e., if the mean value in (2) were zero, we would have (all summations are for all primes $p \leqq N)$

$$
\begin{aligned}
M\left\{\left(f-c_{1}-\right.\right. & \left.\left.\sum c_{p} \varphi_{p}\right)^{2}, N\right\} \\
= & M\left(f^{2}, N\right)-2 \sum c_{p} M\left(f \varphi_{p}, N\right)+c_{1}^{2}-2 c_{1} M(f, N)+\sum c_{p}^{2} \\
= & M\left(f^{2}, N\right)-M^{2}(f, N)-\sum M^{2}\left(f \varphi_{p}, N\right) \\
& +\sum\left(c_{p}-M\left(f \varphi_{p}, N\right)\right)^{2}+\left(c_{1}-M(f, N)\right)^{2} .
\end{aligned}
$$


This is evidently minimized by

$$
c_{1}(f)=M(f(n), N)
$$

and

$$
c_{p}(f)=M\left(f \varphi_{p}, N\right)=p(p-1)^{-1 / 2} M\left(f_{\varepsilon_{p}}, N\right)-(p-1)^{-1 / 2} M(f, N),
$$

or equivalently

$$
c_{p}(f)=p(p-1)^{-1 / 2} N^{-1} \sum_{k=1}^{[N / p]} f(k p)-(p-1)^{-1 / 2} M(f, N) .
$$

These values minimize $d\left(f, F_{N}\right)$ asymptotically, and (11) and (12) will therefore be used to define the coefficients $c_{j}(f), j=1$ or a prime number. This completes the determination of our approximating function $F_{N}(f, n)$ of (3), which differs from a strongly additive function $G_{N}(f, n)$ by a constant only (not depending on $n$ ). Collecting our results, $F_{N}(f, n)$ is defined by the formulas (3), (8), (9), (11) and (12), and its additive part $G_{N}(f, n)$ by (5) and (7). Since our approximation theory can only be appreciated in terms of distribution problems of arithmetical functions, we delay giving examples until the end of the next section.

2. Applications to distribution problems. Let $N v_{N}(n: \cdots)$ denote the number of those integers $n$, not exceeding $N$, for which the property stated in the dotted space holds. For a given arithmetical function $f(n)$, we put

$$
F_{N}(x)=v_{N}(n: f(n)<x),
$$

where $x$ is a real variable. We say that $f(n)$ has a limiting distribution $F(x)$, if $F_{N}(x) \rightarrow F(x)$, for all continuity points of the latter one, as $N \rightarrow$ $+\infty$. Here $F(x)$ is assumed to be a proper distribution function, i.e., increasing, left continuous and at the infinities, its limits are zero and one, respectively. For arithmetical functions $f(n)$ without some specific arithmetical structure, very few results are available to guarantee the existence of a limiting distribution, see [6]. For additive functions, however, there is a (necessary and) sufficient condition for the existence of a limiting distribution (the Erdös theorem [2]). Therefore, by showing that the limiting distributions of an arbitrary $f(n)$ and of its approximation $F_{N}(f, n)$ of the previous section exist simultaneously for a large class of $f(n)$, we shall get a sufficient condition for the existence of $\lim F_{N}(x)$, $N \rightarrow+\infty$, applicable to a large class of functions. Our examples will illustrate the claim that this class of functions is indeed large. The details are as follows.

Let $s(n)$ and $h(n)$ be two arithmetical functions and let $\delta>0$ be an arbitrary positive real number. By simply cancelling those terms in $d(s, h)$ for 
which $|s(n)-h(n)|<\delta$, we immediately have

$$
v_{N}(n:|s(n)-h(n)| \geqq \delta) \leqq \delta^{-2} d(s, h),
$$

which is known in probability theory as the Markov inequality. This inequality (14), combined with the following well known elementary lemma (see, e.g., [7, Lemma 4.1, p. 45]), will be a major step in showing that the distribution of an $f(n)$ can be approximated by that of $F_{N}(f, n)$.

LEMMA 1. Let $s_{N}(n)$ and $h_{N}(n)$ be two sequences of arithmetical functions, such that for any $\delta>0$, as $N \rightarrow+\infty$,

$$
\lim v_{N}\left(n:\left|s_{N}(n)-h_{N}(n)\right| \geqq \delta\right)=0 .
$$

Then $s_{N}(n)$ has a limiting distribution if, and only if, so does $h_{N}(n)$. When they exist, the two limiting distributions coincide.

Writing

$$
f(n)=F_{N}(f, n)+\left\{f(n)-F_{N}(f, n)\right\},
$$

(14) and Lemma 1 immediately yield

LEMMA 2. Let $f(n)$ be an arithmetical function such that, as $N \rightarrow+\infty$, $d\left(f, F_{N}\right) \rightarrow 0$, where $F_{N}(f, n)$ is given in (3). Then $f(n)$ has a limiting distribution if, and only if, $F_{N}(f, n)$ does also. When it exists, the limiting distribution of $f(n)$ coincides with that of $F_{N}(f, n)$.

As we have pointed out, for any $f(n), F_{N}(f, n)$, apart from an additive constant, is a strongly additive function. Namely, from (3) and (5),

$$
F_{N}(f, n)=G_{N}(f, n)+A_{N}
$$

where $G_{N}(f, n)$ is strongly additive and

$$
A_{N}=M(f(n), N)-\sum_{p \leqq N}(p-1)^{-1 / 2} c_{p}(f) .
$$

Note that $A_{N}$ is independent of the variable $n$. In order that the Erdös theorem be applicable in (15), we have to make our final assumptions to enable us to extend the definition of the additive part of (15) to all integers (as it stands, $G_{N}$ is only defined on the integers $1,2, \cdots, N$ ). Assume that $f(n)$ is such that there exist finite real numbers $M(f)$ and $M_{p}(f)$, not depending on $N$, for which

$$
R_{N}(f)=M(f, N)-M(f) \quad \text { and } \quad R_{N}(f, p)=M\left(f \varepsilon_{p}, N\right)-M_{p}(f)
$$

tend to zero. We can thus define a strongly additive function $G(f, n)$, 
independently of $N$, which remains close to $G_{N}(f, n)$. Let

$$
G(f, n)=\sum G(f, p) \varepsilon_{p}(n),
$$

the summation being extended over all primes $p$, where $G(f, p)$ is determined by letting $N$ tend to $+\infty$ in (12), i.e.,

$$
G(f, p)=\{p /(p-1)\}\left\{M_{p}(f) p-M(f)\right\} .
$$

With the technique developed so far, it will now be easy to complete the proof of the following theorem, being our main result.

THEOREM. Let $f(n)$ be an arithmetical function for which each of $d\left(f, F_{N}\right)$ with $F_{N}=F_{N}(f, n)$ given in $(3), R_{N}(f)$ and $R_{N}(f, p)$ of $(17)$ tends to zero as $N \rightarrow+\infty$. Introducing the notation

$$
\begin{aligned}
\|x\|=x & \text { if }|x|<1, \\
& =1 \quad \text { if }|x| \geqq 1,
\end{aligned}
$$

we assume that the series

$$
\sum \frac{\left\|p M_{p}(f)-M(f)\right\|}{p-1} \text { and } \sum \frac{\left\|p M_{p}(f)-M(f)\right\|^{2}}{p-1}
$$

converge, where summations are over all primes. Let further

$$
p R_{N}(f, p)-R_{N}(f)=o\left[(1 / \log \log N)^{1 / 2}\right] .
$$

Under these conditions, $f(n)$ has a limiting distribution.

In view of our development of the general theory on the previous pages, there remain only a few steps to complete the proof. Our assumptions easily yield that in (16), $A_{N}$ has a limit, $A$, say. Lemma 1 and (15) therefore imply that $F_{N}(f, n)$ has a limiting distribution, whenever $G_{N}(f, n)+A$, and thus whenever $G_{N}(f, n)$ itself has. Noting that $G_{N}(f, n)-G(f, n)$ is also strongly additive, our assumption in (21) and the Turán-Kubilius inequality [7, Lemma 3.1a, p. 35] yield

$$
\begin{aligned}
v_{N}\left(n:\left|G_{N}(f, n)-G(f, n)\right| \geqq \delta\right) & \\
& =O\left\{\sum_{p \leqq N} \frac{\left[p R_{N}(f, p)-R_{N}(f)\right]^{2}}{p}\right\}=o(1),
\end{aligned}
$$

as $N \rightarrow+\infty$. In the last step above we made use of the well-known elementary fact that

$$
\sum_{p \leqq N} \frac{1}{p}=O(\log \log N) .
$$


By another appeal to Lemma 1 and by (22) we now have that the limiting distributions of $G_{N}(f, n)$ and of $G(f, n)$ exist simultaneously. Our proof is thus complete, since by (20) and by the Erdös theorem [2] we have that $G(f, n)$ has a limiting distribution, which now implies that $F_{N}(f, n)$ does also. Thus the assumption $d\left(f, F_{N}\right) \rightarrow 0$ and Lemma 2 establish the Theorem.

For historical reasons, we give as our first example, $f(n)=\varphi(n) / n$, where $\varphi(n)$ is the Euler function (this was the first function studied for the existence of limiting distribution). Our conditions are well known to hold, hence the conclusion of our Theorem implies that $\varphi(n) / n$ has a limiting distribution. As a matter of fact, for any multiplicative function $f(n)$ with $|f(n)| \leqq 1$, the condition (20) reduces to the existence of $M(f)$, and when $M(f) \neq 0$, then, in addition, to the convergence of each of the series $\sum\{f(p)-1\} /(p-1)$ and $\sum\{f(p)-1\}^{2} /(p-1)$. When these conditions are satisfied, the other conditions can easily be checked to hold. Hence we get back the sufficiency part of Bakštys' [1] theorem for the special case $|f(n)| \leqq 1$. This latter restriction is not necessary for our purposes; it is however a restriction in most of the studies on the existence of $M(f)$. For the most comprehensive work on $M(f)$ for real valued $f(n)$, see [9]. On the other hand, our Theorem, combined with conditions for $M(f)=0$, implies the corresponding special cases of Galambos [5]. It is interesting to point out that for completely additive functions, $p M_{p}(f)-M(f)=$ $f(p)$, hence our Theorem is a straight extension of the Erdös theorem (the validity of the other conditions easily follows). Other examples are easy to construct and additivity or multiplicativity are evidently unnecessary.

In concluding we remark that it was not essential in our arguments that the variable $n$ should run through the consecutive integers. Whenever the quasi-orthogonality of $\varepsilon_{p}(n)-u_{p}$, with some numbers $u_{p}$, not depending on $n$, remains and if an extension of the Erdös theorem holds, our model provides a similar approximation. Hence, by results of [3], our Theorem can be extended to a general class of sequences of integers as variables. See also the surveys [4] and [8].

I wish to express my sincere thanks to the referee for his careful reading of the manuscript and for his comments.

\section{REFERENCES}

1. A. Bakštys, On the asymptotic distribution of multiplicative number theoretic functions, Litovsk. Mat. Sb. 8 (1968), 5-20. (Russian) MR 40 \#4231.

2. P. Erdös, On the density on some sequences of numbers. III, J. London Math. Soc. 13 (1938), 119-127.

3. J. Galambos, A probabilistic approach to mean values of multiplicative functions, J. London Math. Soc. (2) 2 (1970), 405-419. MR 43 \#176.

4. - Distribution of arithmetical functions. A survey, Ann. Inst. H. Poincaré Sect. B 6 (1970), 281-305. 
5. J. Galambos, On the distribution of strongly multiplicative functions, Bull. London Math. Soc. 3 (1971), 307-312.

6. - - On the asymptotic distribution of values of arithmetical functions, Atti Accad. Naz. Lincei Rend. Cl. Sci. Fis. Mat. Natur. 52 (1972), 126-131.

7. J. Kubilius, Probabilistic methods in the theory of numbers, Gos. Izdat. Polit. Naučn. Lit. Litovsk. SSR, Vilna, 1962; English transl., Transl. Math. Monographs, vol. 11, Amer. Math. Soc., Providence, R.I., 1964. MR 26 \#3691; MR 28 \#3956.

8. - On the distribution of values of arithmetic functions, Trudy Mat. Inst. Steklov. (to appear).

9. E. Wirsing, Das asymptotische Verhalten von Summen über multiplikative Funktionen. II, Acta Math. Acad. Sci. Hungar. 18 (1967), 411-467. MR 36 \#6366

Department of Mathematics, Temple University, Philadelphia, Pennsylvania 19122 